\title{
HOJAS, FLORES Y TALLOS COMESTIBLES NO TRADICIONALES EN COSTA RICA
}

\section{LEAVES, FLOWERS AND EDIBLE STEMS NONTRADITIONAL IN COSTA RICA}

\author{
Francisco Sánchez Montero* \\ Jenny Barrantes Corrales**
}

RESUMEN

64 variedades de hojas, flores y tallos comestibles no tradicionales estudiadas en 14 comunidades del valle central, litorales y fronteras de Costa Rica, aparecen siguiendo líneas isogónicas paralelas al sistema montañoso del país. Existe mayor variedad de hojas durante la estación lluviosa y de flores, durante la seca. Los tallos son perennes todo el año. Motivos culturales, urbanismo y prácticas agrícolas frenan su consumo actual, aunque la población les reconoce gran beneficio para la salud.

PALABRAS CLAVE: ALIMENTOS * PLANTAS SILVESTRES * CONSUMO DE ALIMENTOS NO TRADICIONALES * HOJAS * FLORES * TALLOS

\section{ABSTRACT}

64 varieties of nontraditional edible leaves, flowers and stems were studied in 14 communities of the central valley, coastlines and borders of Costa Rica. These products appear parallel to isogonic lines that follow the mountain system range of the country. There is a greater variety of leaves during the rainy season and of flowers, during the dry season. The stems are perennial all the year. Cultural reasons, urbanism and agricultural practices restrain their present consumption, although the population recognizes their great benefit to health.

KEY WORDS: FOOD * WILD PLANTS * CONSUMPTION OF NON TRADITIONAL FOODS * LEAVES * FLOWERS *SHAFT

Escuela de Nutrición, Universidad de Costa Rica fsmonter@nutricion.ucr.ac.cr
** Escuela de Nutrición, Universidad de Costa Rica jpbarrantes@nutricion.ucr.ac.cr 


\section{INTRODUCCIÓN}

En Costa Rica, la extensa biodiversidad de especies vegetales hizo que tanto sus primitivos habitantes como los que vinieron allende del mar, sobrevivieran $y$ variaran su alimentación gracias al consumo de hojas, flores y tallos que crecían de manera silvestre en las diferentes regiones climáticas del país, tal como lo narran cronistas de esas épocas (Fernández, 1976:370).

Sin embargo, desde el siglo XX con el avance urbanístico, la extensión de la frontera agrícola, que redujo las áreas de cultivo y de foresta (Barrantes y Herrera, 2001:66) y el abuso de tecnologías de producción que afectan el ambiente en exclusivo las partes aéreas de las plantas (Selincourt, 2000), y la transculturación de las costumbres alimentarias que ha experimentado la sociedad a partir del último tercio del siglo pasado, muchos de estos alimentos perdieron protagonismo dada su inaccesibilidad u omisión deliberada de la mesa tradicional.

Esta situación inhabituó al ciudadano a los sabores, olores, colores, texturas y hasta sonidos propios de alimentos sometidos a manipulación doméstica y lo acostumbró a otras características sensoriales y a preparaciones elaboradas industrialmente o de otras culturas (Barrantes y Herrera, 2001:67).

Con el fin de indagar la situación actual de los productos comestibles no tradicionales, se visitaron 14 comunidades ubicadas en diferentes ángulos del país y mediante grupos focales desarrollados con personas identificadas por informantes clave o por diversas asociaciones de la comunidad, como conocedoras de las características, ubicación y formas de preparación de los productos de interés, se logró no sólo identificar $y$ describir estos alimentos, sino también determinar las diferentes formas de su consumo, así como las percepciones populares acerca de estos productos comestibles no tradicionales.

\section{ANÁLISIS DE RESULTADOS}

Las 14 comunidades se ubicaron en el valle central $y$ en las zonas litorales $y$ fronterizas. Participaron de manera voluntaria 137 personas mayores o cercanas a 40 años, residentes antiguas de la zona y aficionadas a preparar alimentos (Cuadro 1, p. 139).

El 52\% de las personas era oriunda de la región central del país, mientras que el $47 \%$, residía en litorales $y$ fronteras, abarcando las 7 provincias. La mayoría habitaba en climas secos $(35 \%)$ y el resto, en climas fríos, templados o cálido-húmedos ( $21 \%$ en cada caso, respectivamente).

Estas personas lograron identificar y describir los productos comestibles no tradicionales de su zona, lo que permitió analizarlos de la siguiente manera.

\section{A. IDENTIFICACIÓN DE HOJAS, FLORES Y TALLOS COMESTIBLES NO TRADICIONALES}

En total se identificaron 64 productos comestibles no tradicionales, de los cuales $56 \%$ (28) correspondió a hojas y 28\% (18) a flores y tallos, respectivamente. El promedio de hojas identificadas por comunidad fue de 7 y de 5 para tallos y flores, cada uno. La identificación fue alta en comunidades del valle central y baja, en las zonas fronterizas y litorales.

En el cuadro 2, se relaciona la mayor o menor identificación de estos productos con la ubicación geográfica de las comunidades en el territorio nacional. Se identifican con sigla mayúscula los alimentos de los cuales se mencionaron más variedades, generalmente entre 8 y 10 especímenes. En sigla cursiva minúscula, se ubican aquellos alimentos de los cuales se mencionaron entre 5 y 7 variedades $y$ finalmente, en sigla minúscula corriente, de los que apenas se dieron entre 2 y 4 ejemplos. Estas frecuencias fueron diferentes para cada especie de alimento ya que por ejemplo, el mayor número de hojas mencionado fue de 10 , pero en el caso de flores y tallos, el número no pasó de 8 .

Se desprende del cuadro (Cuadro 2, p. $140)$ que en el $43 \%(6 / 14)$ de las comunidades participantes, el mayor conocimiento o identificación de productos comestibles no tradicionales corresponde al de hojas, seguido por un conocimiento promedio de flores $(50 \%, 7 / 14) \mathrm{y}$ uno menor, de tallos $(36 \%, 5 / 14)$. 


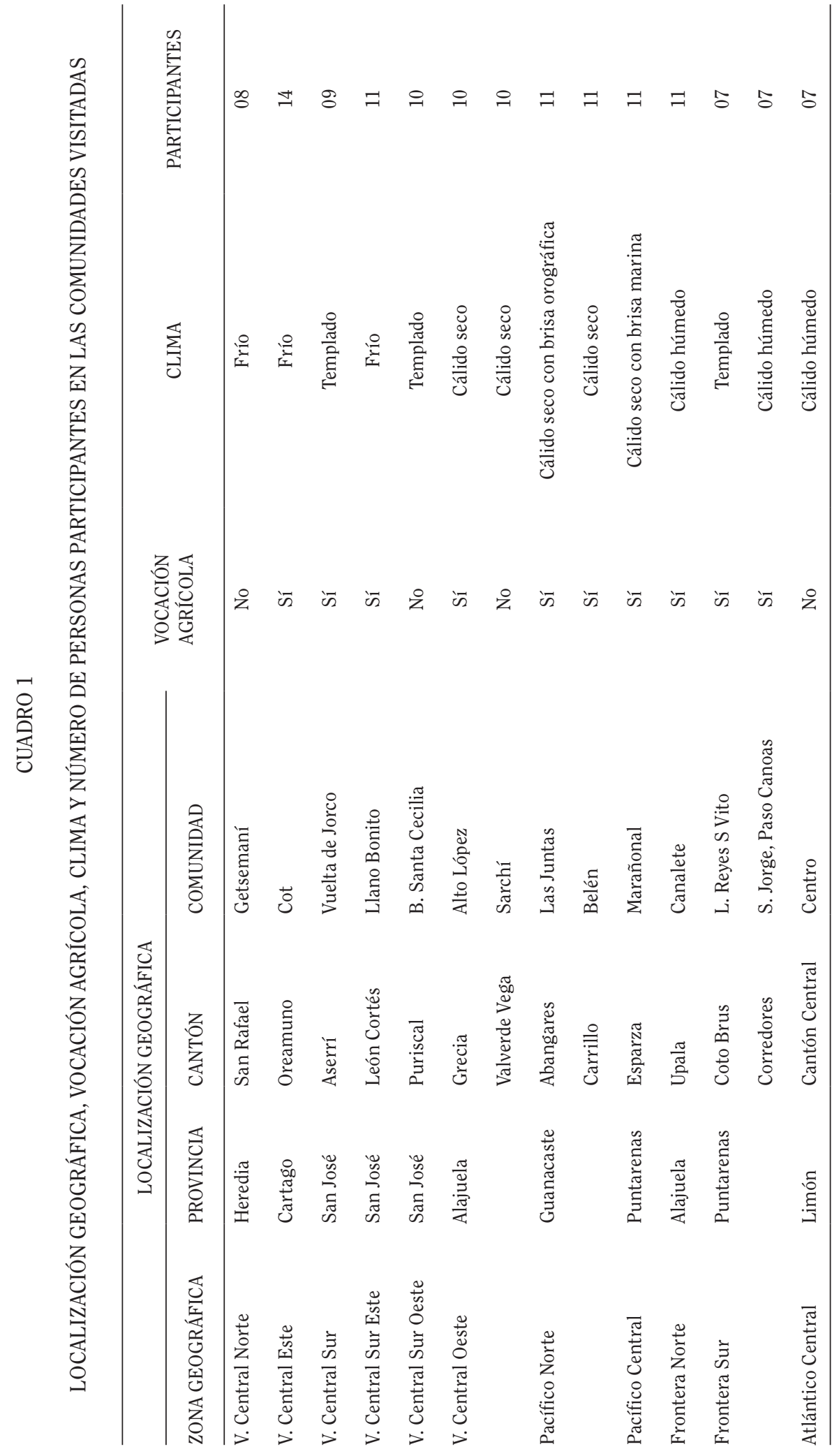




\section{CUADRO 2}

IDENTIFICACIÓN DE LA VARIEDAD DE PRODUCTOS COMESTIBLES NO TRADICIONALES EN LAS COMUNIDADES VISITADAS

\begin{tabular}{|c|c|c|c|c|c|c|c|}
\hline \multirow{2}{*}{\multicolumn{2}{|c|}{ ZONA GEOGRÁFICA }} & \multirow{2}{*}{ PROVINCIA } & \multirow{2}{*}{ CANTÓN } & \multirow{2}{*}{ COMUNIDAD } & \multicolumn{3}{|c|}{ IDENTIFICACIÓN } \\
\hline & & & & & MAYOR & MENOR & MEDIA \\
\hline \multirow{7}{*}{$\begin{array}{l}\text { Valle } \\
\text { Central }\end{array}$} & Norte & Heredia & San Rafael & Getsemaní & $\mathrm{H}$ & $\mathrm{T}$ & $f$ \\
\hline & Sur & San José & Aserrí & Vuelta de Jorco & $\mathrm{HF}$ & $\mathrm{T}$ & \\
\hline & Sur-Este & San José & León Cortés & Llano Bonito & $\mathrm{H}$ & $\mathrm{T}$ & $f$ \\
\hline & Sur-Oeste & San José & Puriscal & B. Sta. Cecilia, Santiago & HT & & $f$ \\
\hline & Este & Cartago & Oreamuno & Cot & $\mathrm{T}$ & $\mathrm{Hf}$ & \\
\hline & \multirow{2}{*}{ Oeste } & \multirow{2}{*}{ Alajuela } & Grecia & Alto López & $\mathrm{F}$ & $\mathrm{T}$ & $h$ \\
\hline & & & Valverde Vega & Sarchí & FT & & $h$ \\
\hline \multirow{3}{*}{ Pacífico } & \multirow{2}{*}{ Norte } & \multirow{2}{*}{ Guanacaste } & Abangares & Las Juntas & $\mathrm{H}$ & & $\mathrm{ft}$ \\
\hline & & & Carrillo & Belén & & Hft & \\
\hline & Central & Puntarenas & Esparza & Marañonal & & & $h f t$ \\
\hline \multirow{3}{*}{ Frontera } & Norte & Alajuela & Upala & Canalete & & & $h f t$ \\
\hline & \multirow{2}{*}{ Sur } & \multirow{2}{*}{ Puntarenas } & Coto Brus & Los Reyes, San Vito & $\mathrm{T}$ & & $h f$ \\
\hline & & & Corredores & San Jorge, Paso Canoas & HFT & & \\
\hline Atlántico & Central & Limón & Cantón Central & Centro & & $\mathrm{Hf}$ & $t$ \\
\hline
\end{tabular}

Nota:

Las siglas H, F o T significan que en esa comunidad se identificó la máxima variedad de hojas, flores o tallos en relación con el total mencionado para cada categoría.

Las siglas h, f o t significan que en esa localidad los ejemplos de hojas, flores o tallos que dieron fueron mínimos según el total encontrado para cada categoría.

Las siglas $h, f$ o $t$ significan que los ejemplos dados estuvieron en el rango medio del total de hojas, flores o tallos identificados en las 14 comunidades en conjunto.

Más específicamente, se nota que mientras en el valle central del país, menos de la mitad de las comunidades participantes, conoce acerca de variedades de tallos comestibles no tradicionales, en las zonas litorales y fronterizas estudiadas, el mayor desconocimiento corresponde al de flores $y$ viceversa, en el centro del país se conocen más hojas y flores y en las fronteras, tallos.
Es probable que este hecho obedezca al clima, por lo que en el cuadro 3 (p. 141) se presenta un resumen de los climas predominantes en los sitios visitados, basado en la clasificación de Holdridge (Tosi, 1969) para Costa Rica.

Se desprende de esta información que en el clima seco tropical es poco probable que se consigan flores y tallos con facilidad, mientras 
CUADRO 3

COMUNIDADES PARTICIPANTES Y PRODUCTO NO TRADICIONAL MÁS CONOCIDO, SEGÚN CLIMA PREDOMINANTE

\begin{tabular}{llll}
\hline COMUNIDAD & PROVINCIA & PRODUCTO MÁS CONOCIDO & CLIMA PREDOMINANTE \\
\hline Belén & Guanacaste & Hojas & Seco tropical transición a húmedo \\
\hline Sarchí & Alajuela & Flores y tallos & Húmedo tropical transición a pre-montano \\
\hline Las Juntas & Guanacaste & Hojas & \\
Marañonal & Puntarenas & Hojas & Húmedo pre-montano \\
Alto López & Alajuela & Flores & \\
\hline Limón & Limón & Tallos & \\
Upala & Alajuela & Hojas & Muy húmedo pre- montano \\
Getsemaní & Heredia & Hojas & \\
Puriscal & San José & Hojas & Húmedo montano bajo \\
San Jorge & Puntarenas & Tallos-hojas & Muy húmedo montano bajo \\
\hline Cot & Cartago & Hojas & Muy húmedo montano \\
\hline Vuelta de Jorco & San José & Hojas & Pluvial pre-montano \\
\hline Llano Bonito & San José & Flores &
\end{tabular}

que en climas con mayor pluviosidad, cercanos o propios de zonas montañosas, si es posible encontrar tales productos con mayor facilidad.

Es así como, en un clima húmedo premontano sería poco probable encontrar tallos, los que se hallarían más frecuentemente en climas muy húmedos pre-montanos, donde por el contrario sería difícil disponer de flores comestibles, las cuales se encontrarían a un nivel superior como el denominado muy húmedo montano. Las hojas tendrían más probabilidad de aparecer en todos los niveles del clima montano bajo, ya fuere húmedo o muy húmedo.

Como se observa, parece que el saber de estas comunidades acerca de los productos comestibles no tradicionales coincide con su asentamiento topográfico, porque al codificarse cada localidad de acuerdo con la variedad de alimento no tradicional predominante, se constató que se conformaban corredores o líneas de mayor o menor disponibilidad de productos, consistentes con las denominadas zonas de vida de Holdridge (Tosi, 1969). Es decir, lugares donde las condiciones eólicas y pluviales determinadas por las montañas, dan origen a diversos suelos, idóneos para determinadas especies vegetales. En el siguiente mapa se demarca el hábitat más favorable para el crecimiento de las hojas, flores y tallos comestibles no tradicionales. 


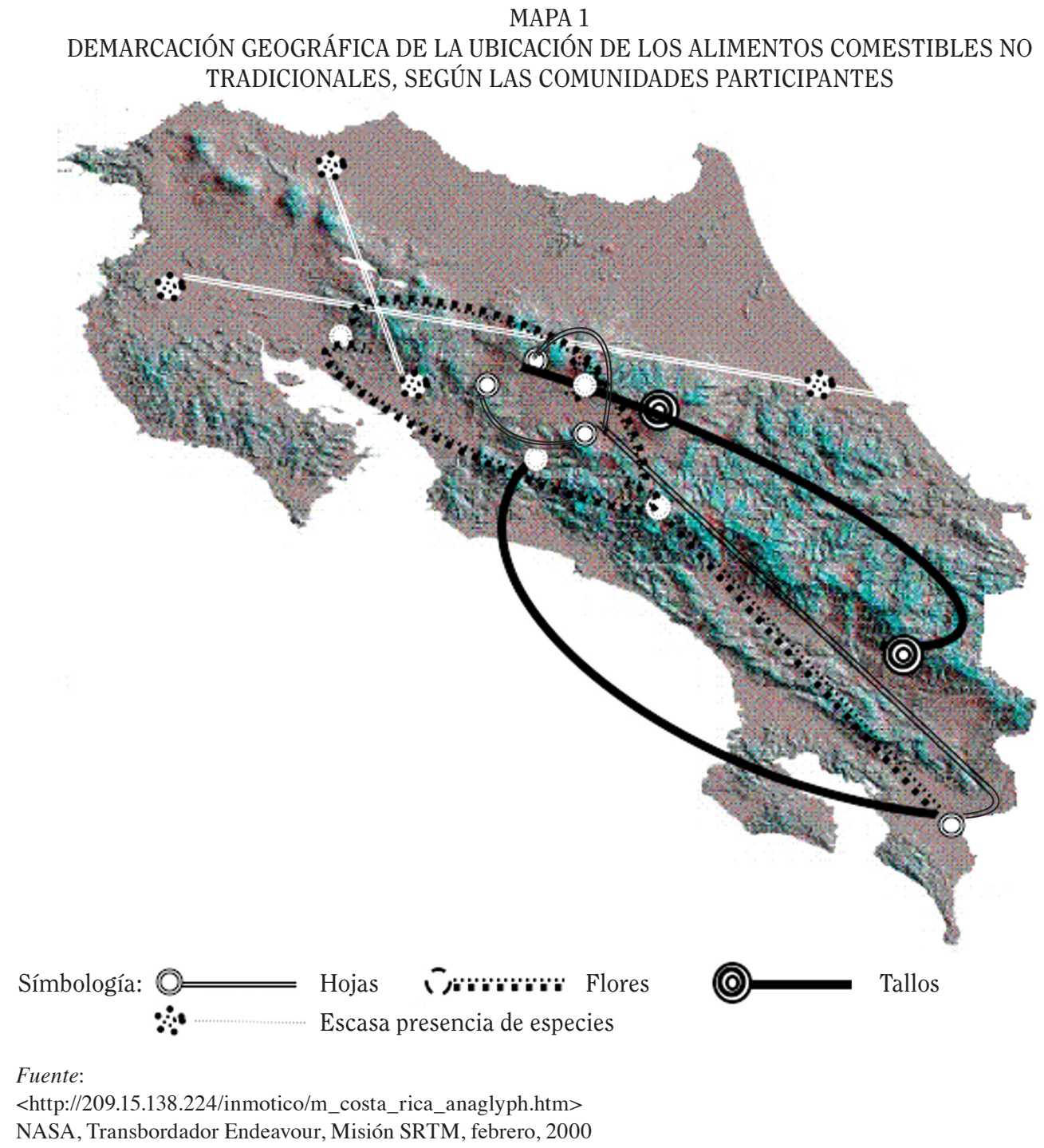

De este mapa se desprende que los alimentos comestibles no tradicionales son más ubicuos en la parte central y sur del país, mientras que escasean al norte $y$ en ambos litorales. Al centro del país se encuentran sitios nucleares donde se amplía la variedad y las formas de preparación conocidas, sobre todo de hojas y flores.

Por otra parte, se confirma que zonas con clima seco no albergan flores ni tallos con facilidad, sino las de climas más húmedos. Las hojas parecen ampararse más que los otros alimentos a las configuraciones montañosas, ya sea en su base, parte media o cima.

B. CARACTERIZACIÓN DE LOS ALIMENTOS COMESTIBLES NO TRADICIONALES

Tanto las características botánicas como nutricionales de estos alimentos se tomaron de la literatura (León y Poveda, 2000; FAO, 1998; Menchú, Méndez, Barrera y Ortega, 1996; Pittier, 1978). Cuando no apareció el producto buscado, se homologó al más cercano dentro de su grupo citado en la literatura.

En cuanto a las características botánicas, se presenta el género representativo, así como algunos ejemplos del mismo. Los géneros de las hojas comprenden alimentos conocidos 
hoy día como hortalizas, cuyas hojas, poca gente consume. Asimismo, los dos grandes géneros botánicos de las flores corresponden a grandes especies vegetales que requieren un amplio terreno disponible, lo cual escapa al modo de vida actual de las personas. Por último, al menos uno de los géneros de tallos estaría más accesible hoy día, dado el consumo de cucurbitáceas que aún perdura, pero se ha perdido la costumbre de comer quelites. El otro género de los tallos, identifica palmeras que crecen en lo profundo de la montaña como el palmito amargo, cuyo consumo es probable que no sea frecuente porque al ser una especie en extinción es patrimonio nacional y su consumo está prohibido.

\section{ESQUEMA 1}

PRINCIPALES GÉNEROS BOTÁNICOS DE LOS ALIMENTOS COMESTIBLES NO TRADICIONALES

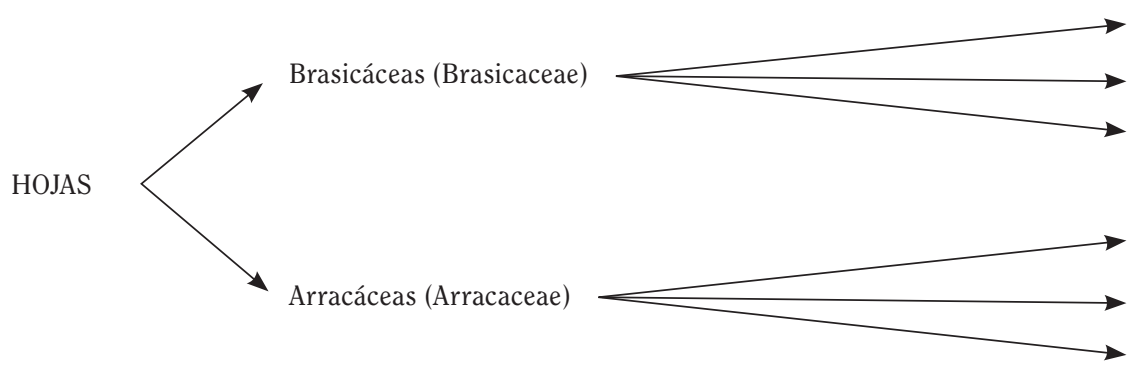

Hojas de rábano

Hojas de coliflor

Hojas de col

Hojas de arracache

Hojas de tiquisque

Hojas de zanahoria

FLORES

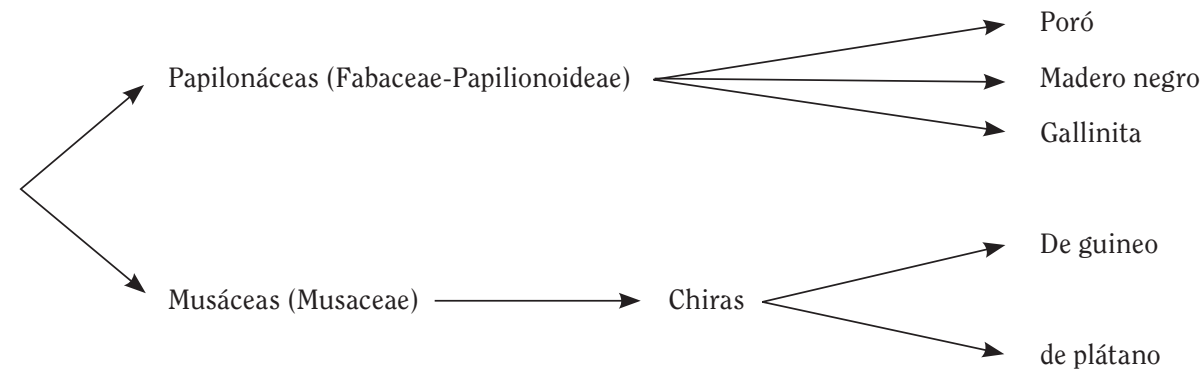

TALLOS

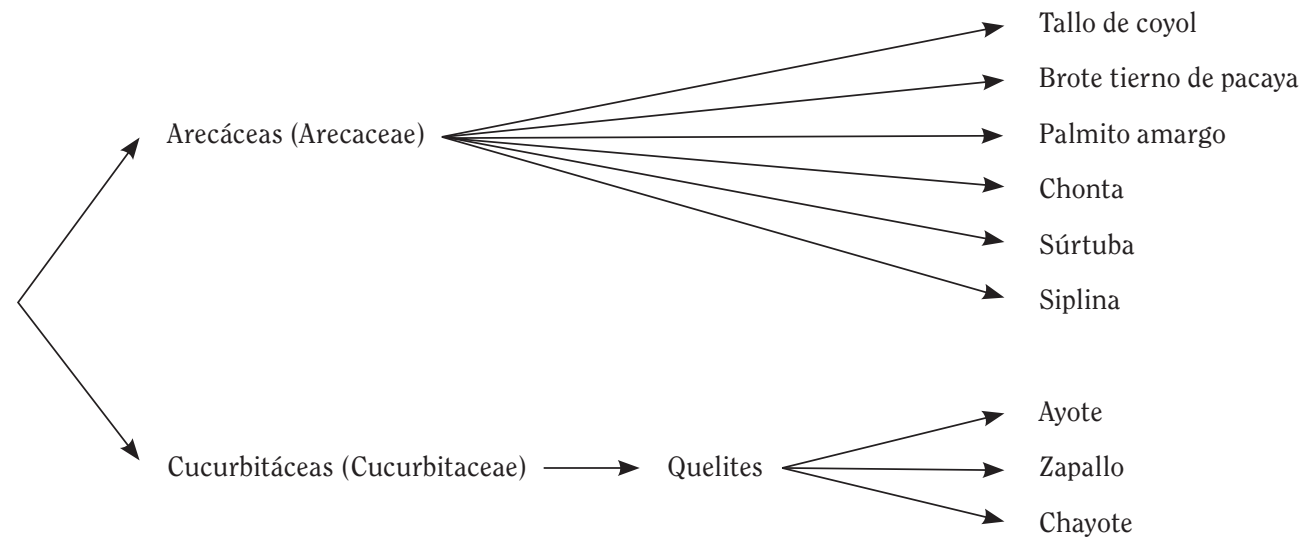


En cuanto al valor nutritivo de estos alimentos, también se recurrió a generalizar, dada la inopia de análisis de las variedades mencionadas por las personas concurrentes a los grupos focales realizados. Aquí se encontró que un mismo alimento recibe diversos nom- bres según la etapa de maduración en que se le encuentre o la forma física que presente. En el esquema siguiente se presentan algunas de las denominaciones ancestrales dadas a estos alimentos; también conocidos como nombres vernáculos.

\section{ESQUEMA 2}

\section{NOMBRES VERNÁCULOS DADOS A LOS PRODUCTOS COMESTIBLES NO TRADICIONALES EN LAS COMUNIDADES PARTICIPANTES}
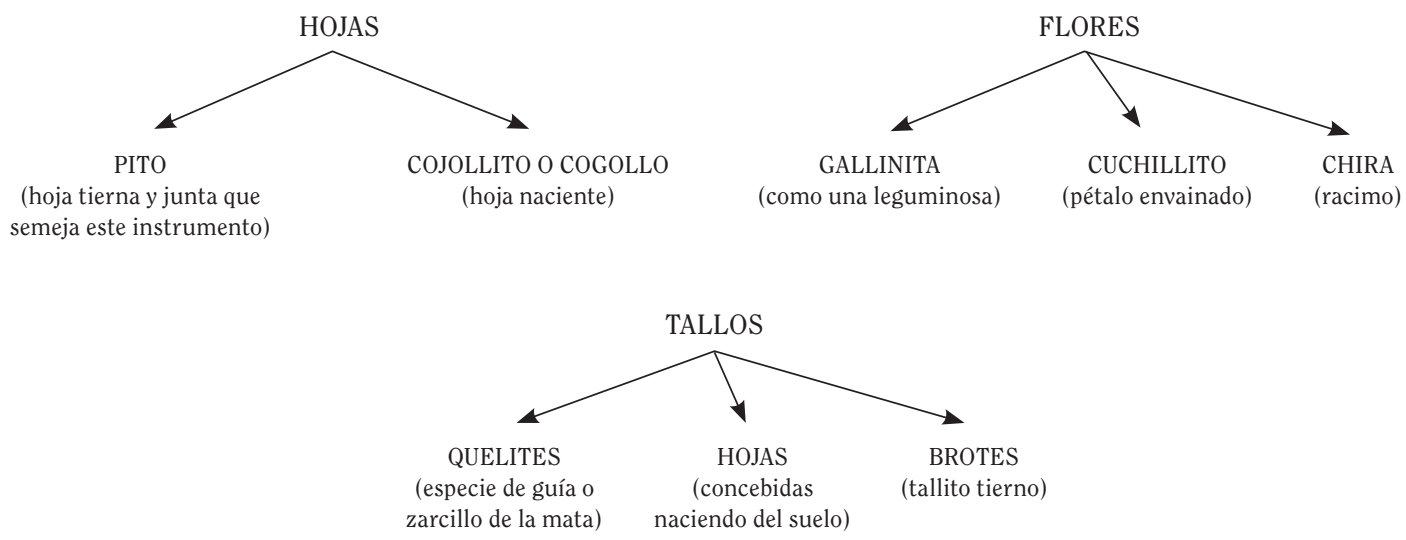

Como se aprecia, cada nombre encierra un amplio concepto que enriquece el aprovechamiento que se le pueda dar al producto. De esta manera, el tallo de una palmera no lo es en el sentido del de los árboles, sino hojas que se entrelazan desde que salen de la tierra. Asimismo, el concepto común de flor con pétalos abiertos, se extiende también al de otras formas no convencionales.

La identificación de diferentes tipos de un mismo alimento, influye en la forma de preparación, porque cada uno se asocia a un estado del desarrollo del vegetal, cambiando así sus características nutricionales y organolépticas.

A falta del análisis específico del valor nutritivo de los diferentes estadíos de las hojas, flores y tallos, sólo se puede especular que las especies más tiernas tendrían mayor concentración de proteínas y grasa, junto con la de algunos minerales como calcio $y$ fósforo $y$ vitaminas como el ácido ascórbico.
Una mayor concentración de carbohidratos, hierro no hemínico y vitaminas del tipo A (concebida en los vegetales como b carotenos), tiamina, riboflavina $y$ niacina son comunes en las especies maduras, que es cuando una parte de la planta, acumula mayor cantidad de nutrientes, convirtiéndose inclusive en fuente de calcio, hierro no hemínico, riboflavina, ácido ascórbico y vitamina A (INCAP-ICNND, 1961:19, $23,25,26,30,32,37,38,40)$.

Sin embargo, de manera general, según la literatura consultada (Menchú, Méndez, Barrera y Ortega, 1996; Maham y EscottStump, 2001), las hojas poseen el mayor cúmulo de los nutrientes mencionados $y$, a diferencia de los tallos, son bajas en energía, lo que las hace ideales para balancear preparaciones que cuenten entre sus ingredientes carnes, huevos o grasas.

En esta escala valorativa, siguen los tallos que aportan mayor concentración de energía 
que las hojas, así como de proteína, fibra y carbohidratos. El menor tenor nutritivo lo ostenta el estado de flor, porque apenas ofrece trazas de grasa, fibra, tiamina, niacina y riboflavina $y$ ningún mineral.

Además, estos alimentos acarrean sustancias químicas o fitonutrientes específicos para determinada función del organismo como el licopeno, flavonoides y terpenos, entre otros, considerados cardio protectores y contrarios al proceso oxidativo o de envejecimiento celular (Tomás-Barberán, 2003: 41-53).

Este último aspecto mencionado es digno de considerar hoy día debido a la prematuridad con que se presentan los procesos degenerativos del organismo humano en las poblaciones (Martínez, Astirian y Madrigal, 2002:175-205).

Una de las formas en que se pueden acelerar, atrasar o eliminar estos procesos es mediante la forma de consumo de los alimentos. En esta investigación se logró recopilar 150 recetas relacionadas con el sistema utilizado por las personas de las comunidades visitadas, de lo cual se expone seguidamente.

\section{FORMAS DE PREPARACIÓN DE LOS ALIMENTOS COMESTIBLES NO TRADICIONALES}

Las técnicas utilizadas para preparar los alimentos no tradicionales oscilan desde el lavado minucioso para despojar a los productos de insectos y suciedad o raspado para eliminar elementos irritantes, hasta otras preparaciones preliminares propias de la receta como picar, pelar, o simplemente desbaratar el producto con la mano. En estos casos se evita sancochar, por ejemplo las hojas, y perder nutrientes solubles en agua, situación contraria a la que ocurre cuando el alimento, una vez cocinado con agua y sal, como la chira de plátano, se lava tantas veces como se quiera para eliminar su sabor amargo, provocando la pérdida de vitaminas hidrosolubles (Barrantes y Herrera, 2001:62).

Los ingredientes utilizados también obedecen a necesidades propias del alimento, por ejemplo, el amargor que poseen muchos de estos productos, se aplaca agregándoles algún otro alimento que contrarreste este sabor, como papa, arracache o musáceas, lo que aumenta además el aporte energético de la preparación (Barrantes y Herrera, 2001: 72). Resta decir el uso universal del huevo y en contadas ocasiones el agregado de carnes como la de res o cerdo (McVicar, 1998:49, 77, 85, 121; de Ortiz, 2004: 88-93).

Con base en estas consideraciones se elaboró un criterio de versatilidad del producto para encontrar la mayor probabilidad de que el alimento fuera consumido, dado a los cambios experimentados al variar su preparación.

Se encontró que la mayor versatilidad la ocuparon las flores, lo cual contrasta con el hecho de que este fue el producto comestible no tradicional menos conocido en las comunidades visitadas, sobre todo las ubicadas en sitios fronterizos y en ambos litorales. Esta paradoja no sucede con las hojas ni tallos donde la gama de productos sí corresponde con la versatilidad de las preparaciones descritas por las personas que participaron en el estudio.

Resulta oportuno mencionar que el conocimiento culinario sobre hojas y flores es mayor en el valle central. Las recetas con tallos abundan más en las zonas fronterizas, especialmente en la del sur. También, tanto en el litoral Pacífico Central como en el del Atlántico, el conocimiento acerca de estos alimentos no tradicionales fue el más deficiente. La versatilidad culinaria de estos productos se presenta en el cuadro 4. (Ver en p. 146).

A fin de hacer más comprensible estas preparaciones se obtuvo un puntaje culinario y nutricional, basado en cinco criterios relacionados con el conocimiento que se tuviera del producto en más de la mitad de las comunidades visitadas, que la preparación tuviese un alto valor nutritivo, ingredientes fácilmente ubicables en los alrededores de la localidad, versatilidad y sencillez de la preparación. El alimento que cumpliera al menos dos criterios, se tomaba como representante del grupo. El cuadro 5 muestra los alimentos que presentaron el puntaje máximo y mínimo. (Ver en p. 147).

Se concluye que las hojas de chicasquil son las que reúnen las cualidades nutricionales y culinarias buscadas y por lo tanto son el alimento sobresaliente de este grupo; así como lo son las flores de itabo $y$ de pacaya para su grupo $y$ el de quelites de ayote y chayote para el 


\section{CUADRO 4}

VERSATILIDAD DEL PRODUCTO, PREPARACIÓN CARACTERÍSTICA Y ALGUNAS FORMAS CULINARIAS DE CONSUMO, ENCONTRADOS EN LAS COMUNIDADES PARTICIPANTES

$\begin{array}{llll}\text { PRODUCTO NO } & \text { VERSATILIDAD DEL } & \text { PREPARACIÓN } & \text { ALGUNAS FORMAS } \\ \text { TRADICIO NAL } & \text { PRODUCTO } & \text { CARACTERÍSTICA } & \text { DE PREPARACIÓN }\end{array}$

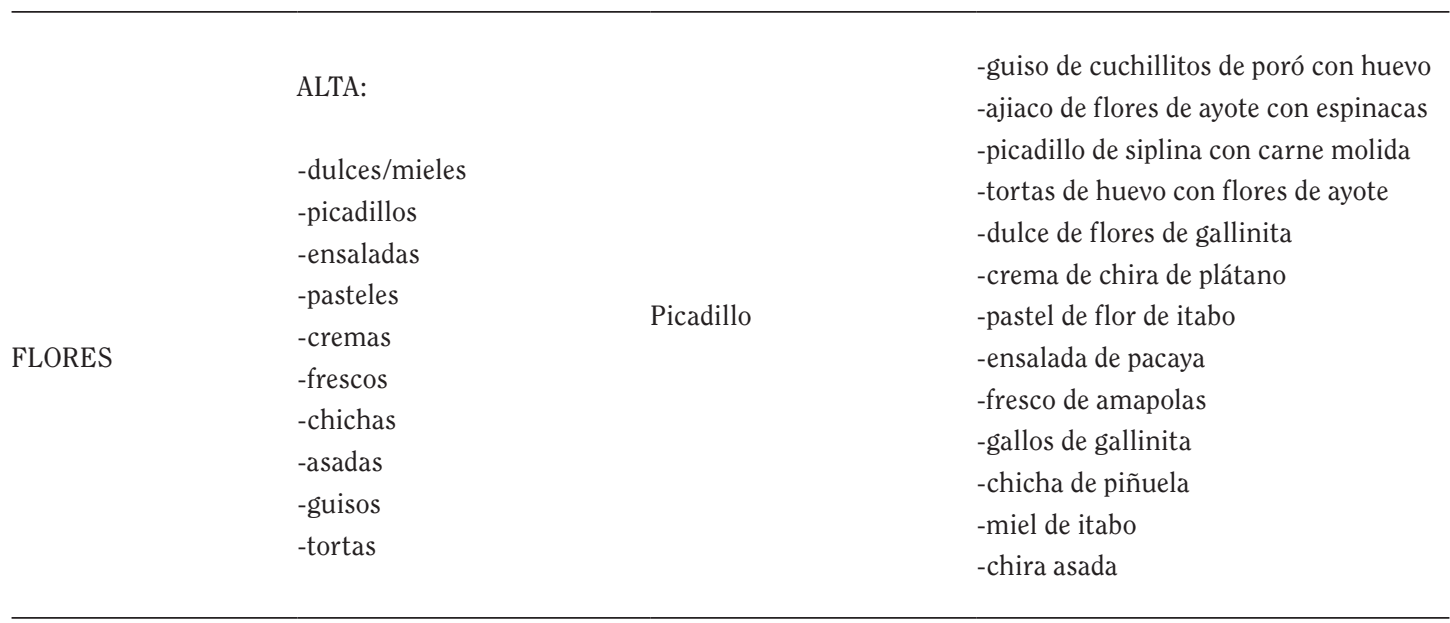

MEDIA:

\begin{tabular}{|c|c|c|c|}
\hline \multirow{8}{*}{ HOJAS } & -albóndigas & \multirow{8}{*}{ Picadillo } & -gallos de zorrillo en varilla fritos \\
\hline & -ensaladas & & -guiso de hojas de rábano \\
\hline & -picadillos & & -arroz con hojas de yuca \\
\hline & -envueltas & & -ensalada de verdolaga \\
\hline & -sofritas & & -sopa de hojas de nabo \\
\hline & -arroces & & -ayaco de chicasquil \\
\hline & -ajiacos & & \\
\hline & -sopas & & \\
\hline
\end{tabular}

BAJA:

-ceviche de palmito de pejibaye -ensalada de tallo de piñuela -sopa de quelites de chayote

TALLOS -picadillos -ensaladas Picadillo -ceviches

-tortas

-sopas -arroz con quelites

-tortas de quelites

-súrtuba asada 


\section{CUADRO 5}

PUNTAJE CULINARIO Y NUTRICIONAL DE LAS HOJAS, FLORES Y TALLOS COMESTIBLES

NO TRADICIONALES, ENCONTRADOS EN LAS COMUNIDADES PARTICIPANTES

\begin{tabular}{|c|c|c|c|c|c|c|}
\hline \multirow{3}{*}{$\begin{array}{l}\text { CRITERIOS NUTRICIONALES } \\
\text { Y CULINARIOS }\end{array}$} & \multicolumn{6}{|c|}{ PUNTAJE } \\
\hline & \multicolumn{3}{|c|}{ MÁXIMO } & \multicolumn{3}{|c|}{ MÍNIMO } \\
\hline & HOJAS & FLORES & TALLOS & HOJAS & FLORES & TALLOS \\
\hline $\begin{array}{l}\text { 1. Producto conocido en más } \\
\text { del } 50 \% \text { de los lugares }\end{array}$ & Verdolaga & $\begin{array}{l}\text { Flor de } \\
\text { itabo }\end{array}$ & $\begin{array}{l}\text { Quelites de } \\
\text { ayote }\end{array}$ & Tiquisque & $\begin{array}{l}\text { Flor de la } \\
\text { piñuela }\end{array}$ & $\begin{array}{l}\text { Brote tierno de } \\
\text { pacaya }\end{array}$ \\
\hline 2. Alto valor nutritivo & Chicasquil & Pacaya & $\begin{array}{l}\text { Brote tierno } \\
\text { de pacaya }\end{array}$ & Verdolaga & Flor de ayote & Tallo de pejibaye \\
\hline $\begin{array}{l}\text { 3. Fácil obtención en la } \\
\text { localidad }\end{array}$ & Chicasquil & Chira & Bambú & $\begin{array}{l}\text { Hojas de } \\
\text { rábano }\end{array}$ & Madero negro & Quelites de yuca \\
\hline $\begin{array}{l}\text { 4. Versatilidad en la } \\
\text { preparación }\end{array}$ & Chicasquil & $\begin{array}{l}\text { Flor de } \\
\text { itabo }\end{array}$ & $\begin{array}{l}\text { Quelites de } \\
\text { chayote }\end{array}$ & Hojas de col & Pacaya & Quelites de yuca \\
\hline 5. Facilidad en la preparación & Verdolaga & Pacaya & Palmito & Hojas de yuca & $\begin{array}{l}\text { Flor de la } \\
\text { piñuela }\end{array}$ & Quelites de yuca \\
\hline
\end{tabular}

conglomerado de tallos. Los alimentos que no alcanzaron notabilidad en su aspecto nutricional ni culinario fueron la flor de la piñuela y los quelites de yuca.

Como es sabido, dichas preparaciones están sujetas a que el producto esté disponible, por esta razón en el cuadro 6 se muestra su estacionalidad.

Se observa que en las comunidades de la zona central del país existe mayor probabilidad de conseguir hojas comestibles no tradicionales todo el año y con mayor variedad durante la estación lluviosa. Por su parte, los tallos tampoco muestran ningún cambio estacional, siendo perennes todo el año. Las flores por su parte, están más influenciadas por la estacionalidad, encontrándose ligeramente más especímenes durante la estación seca.

En las comunidades participantes de los litorales $y$ fronteras, en términos generales, los tallos también muestran presencia todo el año $y$ poco efecto de la estacionalidad, no así las hojas y las flores que dependen de la época del año que se trate.

Una vez conocido el cómo se utilizan los alimentos comestibles no tradicionales, es necesario explorar las razones por las cuales los habitantes los han relegado a las últimas opciones de su menú habitual, lo cual se presenta en el siguiente apartado. 


\section{CUADRO 6}

\section{ESTACIONALIDAD DE ALGUNOS ALIMENTOS COMESTIBLES NO TRADICIONALES SEGÚN ZONA GEOGRÁFICA}

\begin{tabular}{|c|c|c|c|c|c|}
\hline ZONA GEOGRÁFICA & ALIMENTOS & ESTACIÓN SECA & ESTACIÓN LLUVIOSA & TOI & EL AÑO \\
\hline \multirow[t]{3}{*}{$\begin{array}{l}\text { Zona central } \\
\text { del país }\end{array}$} & Hojas & & $\begin{array}{l}\text {-chicasquil } \\
\text {-zorrillo } \\
\text {-zapallo } \\
\text {-ayote }\end{array}$ & $\begin{array}{l}\text {-hojas de } \\
\text { zanahoria } \\
\text {-chicasquil }\end{array}$ & $\begin{array}{l}\text {-hojas de coliflor } \\
\text {-hojas de ortiga }\end{array}$ \\
\hline & Flores & $\begin{array}{l}\text {-piñuela } \\
\text {-itabo } \\
\text {-chiverre }\end{array}$ & -flor de pacaya & $\begin{array}{l}\text {-chira } \\
\text {-poró }\end{array}$ & -amapola \\
\hline & Tallos & & & $\begin{array}{l}\text {-chayote } \\
\text {-ayote } \\
\text {-papaya } \\
\text {-súrtuba }\end{array}$ & $\begin{array}{l}\text {-puntas de zapallo } \\
\text {-brotes de bambú }\end{array}$ \\
\hline \multirow[t]{3}{*}{$\begin{array}{l}\text { Litorales } \\
y \text { fronteras }\end{array}$} & Hojas & $\begin{array}{l}\text {-zorrillo } \\
\text {-chicasquil }\end{array}$ & -hojas en general & -rábano & \\
\hline & Flores & -flor de itabo & -flor de ayote & -chiras & \\
\hline & Tallos & & & $\begin{array}{l}\text {-quelites de } \\
\text { chayote } y \text { ayote }\end{array}$ & -bambú \\
\hline
\end{tabular}

D. FACTORES QUE AFECTAN LA UTILIZACIÓN DE LOS ALIMENTOS NO TRADICIONALES

Mediante la técnica de análisis de aspectos fortalecedores o debilitantes, así como de aquéllos posibles de potenciar (oportunidades) o inhibir (amenazas) una situación dada, se logró completar en la siguiente matriz, cuya información se extrajo de los grupos focales realizados en las 14 comunidades.

MATRIZ

ANÁLISIS DE SITUACIONES REALES O POSIBLES QUE AFECTAN LA UTILIZACIÓN DE LOS ALIMENTOS COMESTIBLES NO TRADICIONALES EN LAS COMUNIDADES VISITADAS

\begin{tabular}{ll}
\hline \multicolumn{1}{c}{ SITUACIONES POSITIVAS REALES (FORTALEZAS) } & \multicolumn{1}{c}{$\begin{array}{c}\text { POSIBLES SITUACIONES POSITIVAS } \\
\text { (OPORTUNIDADES) }\end{array}$} \\
\hline - intercambio de productos entre vecinos & $\begin{array}{c}\text { comercialización de estos productos en } \\
\text { comunidades vecinas }\end{array}$ \\
- reconocimiento de un valor nutritivo superior al de un & - interés en la población por rescatar el consumo \\
alimento procesado & de estos alimentos \\
- transmisión generacional del consumo de estos & - utilización en festividades comunales \\
productos no tradicionales & - auge de la agricultura orgánica \\
- venta en negocios de la comunidad & - uso de estos productos como plantas \\
- presencia libre en cafetales, cercas y potreros & ornamentales comestibles \\
- cultivos en patios o fincas &
\end{tabular}


MATRIZ (Continuación)

ANÁLISIS DE SITUACIONES REALES O POSIBLES QUE AFECTAN LA UTILIZACIÓN DE LOS ALIMENTOS COMESTIBLES NO TRADICIONALES EN LAS COMUNIDADES VISITADAS

\begin{tabular}{ll}
\hline \multicolumn{1}{c}{ SITUACIONES NEGATIVAS REALES (DEBILIDADES) } & POSIBLES SITUACIONES NEGATIVAS (AMENAZAS) \\
\hline - desconocimiento de la reproducción de estos productos & - estilos de vida que privilegian las preparaciones \\
por crecer libremente en veredas, caminos o cercas del & rápidas a base de carne \\
campo & - trabajo remunerado de la mujer fuera del hogar \\
- hurto de estos productos en el vecindario & - temor acerca de la procedencia de los productos \\
- urbanización del campo & comestibles no tradicionales \\
- uso incontrolable de agroquímicos & - campañas educativas en salud desacreditan \\
- prácticas agrícolas inadecuadas (chapeo mecanizado) & alimentos base para estas preparaciones, como el \\
- desvalorización de la vida campesina & - asociación de estos productos con la pobreza \\
- falta de carácter en la educación de los hijos & extrema de los ancestros \\
- propaganda comercial parcializada hacia un tipo de & \\
alimentación & \\
\hline
\end{tabular}

Las fortalezas y debilidades indican situaciones reales sobre las cuales se podría intervenir para reforzarlas o mejorarlas en un caso, o en el otro, asumir las debilidades como retos para incentivar el seguimiento de estrategias creativas como podría ser aprovechar el sistema educativo y de salud del país para realizar campañas que destaquen el valor tanto nutricional como cultural de estos alimentos cada vez más relegados por un mal entendido desarrollo social.

Situación inversa juegan las oportunidades $y$ amenazas que afectan a estos productos no tradicionales, porque son situaciones que dependen de muchas circunstancias para hacerse realidad. Por ejemplo, la asociación de estos productos con la pobreza extrema de los mayores, es un fantasma que se hace presente, entre otros, porque no existe un orgullo por los valores nacionales. Si no se estimulan estos, la situación negativa pasaría de amenaza a un hecho real, como podría ser el somatizar o sentir náuseas u otras barreras psicológicas por el solo recuerdo de estos alimentos.

A fin de aclarar estos conflictos hallados en las comunidades visitadas, se ahondó en las percepciones que aún manifiesta la población en relación con los alimentos de interés.

\section{E. PERCEPCIONES ACERCA DE LOS PRODUCTOS COMESTIBLES NO TRADICIONALES}

Del análisis cualitativo de las transcripciones de los grupos focales se logró extraer categorías generales acerca del por qué estos alimentos desaparecieron o están en franco retroceso en la dieta nacional.

Se encontró que la percepción, entendida como un proceso mediante el cual la persona conscientemente adquiere conocimiento de la realidad, se focalizaba hacia cuatro aspectos relacionados con la explicación que daban las personas de las comunidades participantes, sobre el desuso de estos alimentos. Estas razones giraron en torno a la disponibilidad de los productos no tradicionales, su modo de preparación, barreras en el consumo y beneficios de estos alimentos. El mapa conceptual de la siguiente página resume esta situación.

Es necesario aclarar que en este mapa conceptual se contempló la categoría de "beneficios" que no calza con el sentido del discurso aquí presentado pero que se creyó oportuno incluir porque las personas hacían una especie de reflexión o auto reprensión, posiblemente por influencia de los autores de este artículo que se presentaron como nutricionistas interesados en 
recopilar información sobre alimentos no utilizados actualmente pero que era muy importante conocerlos para ayudar a la salud.

Fuera de esta categoría, las restantes giran en torno a tres aspectos justificables en cierta medida por el inevitable desarrollo social de las comunidades y cuyo cambio requeriría construir un verdadero carácter nacional que lograse armonizar el avance de los pueblos con la estima del origen labriego del pueblo costarricense y de su simbiosis con el medio natural del país.
Sin embargo, entre las razones dadas para cada uno de los tres aspectos mencionados, las relacionadas con la dificultad para la obtención del alimento corresponden a factores externos difíciles de revertir; no tanto las asociadas con las formas y barreras en su consumo, porque caen dentro del campo mental y subjetivo de la creencia, señalada como algo común en las generaciones más jóvenes y por lo tanto posible de modificar con acciones socio-educativas diseñadas especialmente para este grupo etáreo.

\section{MAPA CONCEPTUAL}

\section{PERCEPCIÓN ACERCA DE LOS ALIMENTOS COMESTIBLES NO TRADICIONALES}

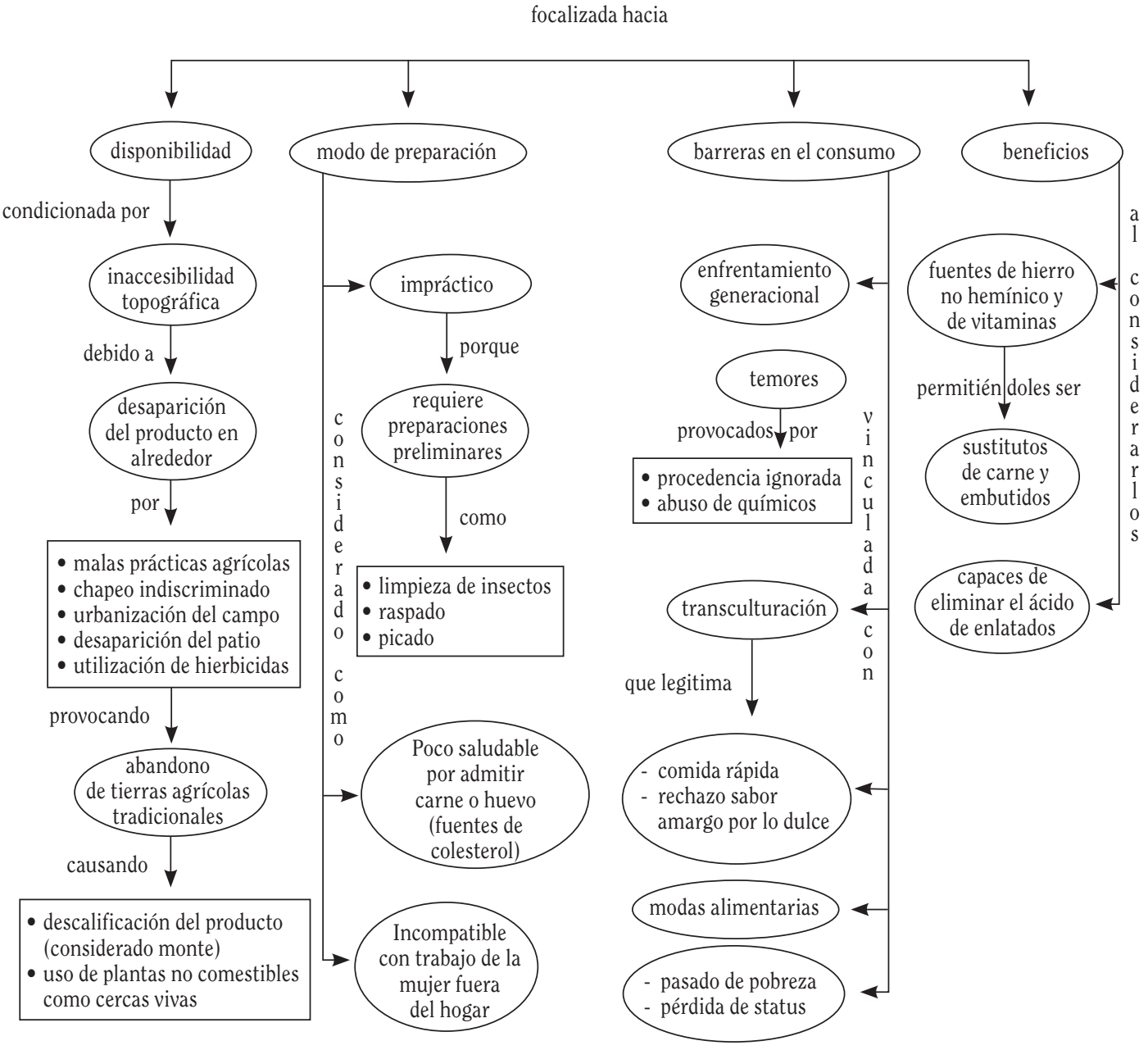

Fuente: Elaboración propia basada en el análisis de grupos focales realizados en 14 comunidades. 


\section{DISCUSIÓN Y CONCLUSIONES}

Para un país que dado su aislamiento social y político de los siglos pasados, superó muchas de sus carencias alimentarias con base en una dieta de productos de la tierra (Fernández, 1976:370), identificar vegetales no tradicionales, conlleva un significado no sólo antropológico, sino también de salud pública, porque de igual forma que sustentaron la vida de los ancestros, aportándoles sustancias nutritivas esenciales para el trabajo y el aprendizaje, de igual manera, hoy día podrían ser uno de los factores indispensables en la prevención de diversas enfermedades crónicas y degenerativas que afectan a la comunidad nacional (Hernández y León, 1992:21-22; Martínez, Asterian y Madrigal, 2002:175-2005).

También, se podría vincular la importancia de haber identificado estos alimentos con el desarrollo de los lugares donde se encontraron, porque existe una tendencia mundial a volver al consumo de hojas, flores y tallos silvestres, dados sus probados beneficios, especialmente para balancear el consumo excesivo de alimentos industrializados que prevalece hoy día.

Por otra parte, debido a que una de las principales fuentes de ingresos en todo el territorio nacional es el turismo, $y$ en este estudio se han identificado verdaderos corredores naturales de hojas, flores y tallos, las comunidades podrían potenciar este hecho y poner en práctica las 150 recetas que se obtuvieron de este estudio y cuyo factor gastronómico, basado en experiencia de otros países (McVicar, 1998; De Ortiz, 2004) será objeto de otro análisis.

Por el momento, resulta valioso saber que con sencillas prácticas de cultivo más armónicas con el ambiente y la vuelta a los patios tradicionales, cualquier familia campesina podría mejorar su salud y su nivel de ingreso. Se destaca el ámbito rural porque, llama la atención que es en estas zonas donde existe el menor consumo de vegetales (Ministerio de Salud, 2003).

Un elemento que podría guiar futuras intervenciones en pro del cultivo y consumo de alimentos como los descritos en este trabajo, es tomar en cuenta el elenco de fortalezas y debilidades reales y las figuradas como oportunidades $y$ amenazas en las comunidades. Para esta eventualidad, no sobraría tomar en cuenta los diversos géneros botánicos que sobresalieron en esta investigación, ni tampoco repasar las variadas formas de preparación que permitieron asignar un puntaje culinario $y$ nutricional al consumo de los productos encontrados.

En suma, investigar alimentos comestibles no tradicionales, podría ser un asunto de agenda permanente para capitalizar la gran diversidad vegetal que podría perderse, si no se valoran y estudian más las características de estos alimentos y se les reconocen sus múltiples beneficios, entre ellos, que pueden ser un medio de apoyo para la seguridad alimentaria y nutricional al ser especies locales que pueden ofrecer un mejor aprovechamiento porque presentan costos de aclimatación reducidos, reproducción local y adaptación total al contexto geográfico (Hernández y León, 1992:4-11).

Con esta investigación se ha podido constatar que aún existe en el país conocimiento acerca de algunos alimentos comestibles no tradicionales. Dicho conocimiento se basa en la capacidad de identificar 64 variedades de estos productos, sobre todo de hojas $y$ en segundo lugar, flores y tallos, en igual número de especimenes, respectivamente. Enriquece este saber, el hecho de que se conocen 74 formas de preparar las hojas; 42, los tallos y 34, las flores, no presentadas en este trabajo por corresponder a otro tipo de análisis.

Con base en esta particularidad, se concluye también, que uno de los hallazgos más importantes, fue demostrar que las distintas variedades de estos productos siguen un itinerario geográfico lógico puesto que se presentan de acuerdo con la orientación orográfica del territorio nacional. Esto permitió saber que el régimen de lluvias $y$ vientos $y$ la altitud son factores que potencian o adversan el crecimiento de estos alimentos no tradicionales.

Por último, se concluye que, a pesar de la gama de preparaciones descrita, hay dificultad en la obtención de estos alimentos por diversas razones relacionadas con factores propios del desarrollo agrario nacional, la forma de consumo de hojas, flores y tallos, que requieren ciertas precauciones preliminares demandantes de tiempo y la existencia de barreras mentales para su consumo. 


\section{BIBLIOGRAFÍA}

Barrantes, J. y Herrera, L. "Disponibilidad y consumo de las flores, tallos y hojas comestibles no tradicionales en tres comunidades del Valle Central de Costa Rica”. [Tesis de Licenciatura en Nutrición]. Escuela de Nutrición UCR, 2001.

De Ortiz M, GP. Delicias gastronómicas. $1^{\mathrm{a}}$. Ed. Bogotá: Fondo de Cultura, 2004.

FAO. Valor nutritivo y usos en alimentación humana de algunos cultivos autóctonos subexplotados de Mesoamérica. Santiago, Chile: AO, 1993.

Fernández, L. Asentamientos, hacienda y gobierno (Nicoya, el Golfo, Cartago y Valle Central). Gobierno y Constitución 3. San José: Editorial Costa Rica, 1976.

Hernández B, J.E. y León, J.(Editores). Cultivos marginados: otra perspectiva de 1492. Colección FAO. Jardín Botánico de Córdoba. Roma: FAO, 1992.

León, J. y Poveda, L.J. Nombres comunes de las plantas en Costa Rica. 1 $1^{a}$. Edición. San José, Costa Rica: Editorial Guayacán, 2000 .

Pittier, H. Plantas usuales de Costa Rica. San José: Editorial Costa Rica, 1978.

Maham, K. y Escott-Stump, S. Nutrición y dietoterapia de Krause. 10a. Ed. México: McGraw-Hill Interamericana, 2001.
Martínez, J.A.; Astiarán, I. y Madrigal, H. Alimentación y Salud Pública. 2a. Ed. Madrid: McGraw-Hill Interamericana, 2002.

McVicar, J. Del jardín a la mesa. Barcelona. Tres Torres: Edunsa, 1998.

Menchú, M.T. Méndez, H.; Barrera, H. y Ortega, L. Tabla de composición de alimentos para Centro América. Guatemala: INCAP, 1996.

Ministerio de Salud. Encuesta nacional de consumo de alimentos. Encuesta de Hogares y Propósitos Múltiples, 2001. Costa Rica: Ministerio de Salud, 2003.

Selincourt, K. "Medio Ambiente y Recursos Naturales". Revista Agricultura de las Américas [en línea] Colombia, junio, 2000. En: <www_sica_gov_ec agro negocios $>$ [Consulta: 11 setiembre, 2001].

Tomás-Barberán, F.A. "Los polifenoles de los alimentos y la salud". Alim. Nutri. Salud 10 (2). 2003: 41-53.

Tosi, J. Mapa ecológico. Según la clasificación de zonas de vida del mundo del L.R. Holdridge. San José, Costa Rica: Centro Científico Tropical, 1969.

Wu Leung, W-T y Flores, M. Tabla de composición de alimentos para uso en América Latina. Guatemala: INCAP-ICNND, 1961. 